\title{
Reflex Responses in Plasma Catecholamines Caused by Static Contraction of Skeletal Muscle
}

\author{
Kanji MATSUKAWA, Tomoko SADAMOTO*, Hirotsugu TSUCHIMOCHI, \\ Hidehiko KOMINE, Jun MURATA, and Kiyoshi SHIMIZU \\ Department of Physiology, Institute of Health Sciences, \\ Hiroshima University Faculty of Medicine, Hiroshima, 734-8551 Japan; and \\ *Human Behavioral Sciences, Division of Integrated Sciences, Graduate School of Human Culture, \\ Nara Women's University, Nara, 630-8526 Japan
}

\begin{abstract}
To examine a hypothesis of whether static muscle contraction produces a release of catecholamines from the adrenal medulla via reflex stimulation of preganglionic adrenal sympathetic nerve activity induced by receptors in the contracting muscle, we compared the reflex responses in a concentration of epinephrine (Ep) and norepinephrine (NEp) in arterial plasma during static contraction and during a mechanical stretch of the hindlimb triceps surae muscle in anesthetized cats. Static contraction was evoked by electrically stimulating the peripheral ends of the cut $L_{7}$ and $S_{1}$ ventral roots at 20 or $40 \mathrm{~Hz}$. Mean arterial pressure (MAP) and heart rate $(\mathrm{HR})$ increased $23 \pm 3.1 \mathrm{mmHg}$ and $19 \pm 4.3$ beats/min during static contraction. Ep in arterial plasma increased $0.18 \pm 0.072 \mathrm{ng} / \mathrm{ml}$ over the control of $0.14 \pm 0.051 \mathrm{ng} / \mathrm{ml}$ within $1 \mathrm{~min}$ from the onset of static contraction, and NEp increased $0.47 \pm 0.087 \mathrm{ng} / \mathrm{ml}$ over the control of $0.71 \pm$
\end{abstract}

$0.108 \mathrm{ng} / \mathrm{ml}$. Following a neuromuscular blockade, although the same ventral root stimulation failed to produce the cardiovascular and plasma catecholamine responses, the mechanical stretch of the muscle increased MAP, HR, and plasma Ep, but not plasma NEp. With bilateral adrenalectomy, the baseline Ep became negligible $(0.012 \pm 0.001 \mathrm{ng} / \mathrm{ml})$ and the baseline NEp was lowered to $0.52 \pm 0.109 \mathrm{ng} / \mathrm{ml}$. Neither static contraction nor mechanical stretch produced significant responses in plasma Ep and NEp following the adrenalectomy. These results suggest that static muscle contraction augments preganglionic adrenal sympathetic nerve activity, which in turn secretes epinephrine from the adrenal medulla into plasma. A muscle mechanoreflex from the contracting muscle may play a role in stimulation of the adrenal sympathetic nerve activity. [Japanese Journal of Physiology, 51, 591-597, 2001]

Key words: norepinephrine, epinephrine, adrenal medulla, adrenal sympathetic nerve activity, mechanical stretch of muscle.

Muscle contraction activates small muscular afferents (myelinated group III and unmyelinated group IV fibers), which is believed to initiate autonomic reflex adjustment of the cardiovascular system. It was demonstrated that sympathetic outflows to the heart and the kidneys increase during static muscle contraction induced by electrical stimulation of the ventral roots in anesthetized cats [1-4]. The increase in car- diac sympathetic nerve activity correlates with an increase in heart rate [3], and the increase in renal sympathetic nerve activity correlates with reduced renal blood flow [2], suggesting that the reflex changes in postganglionic sympathetic nerve activities caused by skeletal muscle contraction contribute to cardiovascular adjustments, such as increases in cardiac output and arterial blood pressure and a redistribution of car-

Received on May 21, 2001; accepted on July 10, 2001

Correspondence should be addressed to: Kanji Matsukawa, Department of Physiology, Institute of Health Sciences, Hiroshima University Faculty of Medicine, Kasumi 1-2-3, Minami-ku, Hiroshima, 734-8551 Japan. Tel: +81-82-257-5435, Fax: +81-82-257-5439, E-mail: matsuk@hiroshima-u.ac.jp 
diac output.

Furthermore, if preganglionic sympathetic nerve activity to the adrenal medulla (AdSNA) is reflexly stimulated during muscle contraction, catecholamines secreted into plasma from the adrenal medulla may develop a slower but more widespread and sustained cardiovascular response in concert with the direct effect of postganglionic sympathetic nerve activity. Recently, the reflex response in AdSNA to static muscle contraction was recorded in anesthetized rats [5]. AdSNA increased during muscle contraction induced by electrical stimulation of the tibial nerve. Thus it is very likely that the increase in AdSNA contributes to a release of catecholamines from the adrenal medulla. However, the reflex changes in the concentration of plasma epinephrine and norepinephrine in response to static muscle contraction remained unknown.

Although both types of small muscular afferents (myelinated group III and unmyelinated group IV fibers) are activated during muscle contraction, the characteristics of the recruitment pattern are different in time course and magnitude [6]. Myelinated group III fibers are quite likely to be excited by a mechanical event during muscle contraction, and unmyelinated group IV fibers are quite likely to be excited by a metabolic byproduct released during muscle contraction and/or a change in muscular interstitial environment. Since the passive mechanical stretch of muscle in a physiological range can stimulate myelinated group III afferents rather than unmyelinated group IV afferents, a comparison in the reflex responses of sympathetic efferent nerve activity between the contraction and the mechanical stretch of skeletal muscle should lead to an insight into the afferent mechanisms in the contracting muscle. The mechanical stretch of muscle increases cardiac and renal sympathetic nerve activities to 85 and $51 \%$ of their responses observed during static contraction with the same tension development $[1,3]$. Regarding AdSNA, it increased immediately after the onset of static muscle contraction in anesthetized rats [5]. The result suggests that the passive mechanical stretch of muscle also can stimulate AdSNA, which in turn secretes epinephrine from the adrenal medulla into plasma. However, the reflex response in plasma epinephrine to passive stretch and to static muscle contraction has not been studied.

We hypothesized that AdSNA is augmented reflexly not only by muscle contraction, but also by mechanical stretch, which contributes to a release of catecholamines from the adrenal medulla. To examine this hypothesis, we compared the reflex responses in a concentration of plasma catecholamines to static contraction and mechanical stretch of the hindlimb triceps surae muscle in anesthetized cats. Static contraction was evoked by electrically stimulating the peripheral ends of the sectioned $\mathrm{L}_{7}$ and $\mathrm{S}_{1}$ ventral roots.

\section{METHODS}

Preparations of animals. The present study was conducted on 15 anesthetized cats (body weight 2.6-4.3 kg) under the Guiding Principles for the Care and Use of Animals in the Fields of Physiological Sciences approved by the Physiological Society of Japan. Each animal was anesthetized by the inhalation of a gas mixture of $4 \%$ halothane, nitric oxide $\left(\mathrm{N}_{2} \mathrm{O}\right)$, and oxygen. An endotracheal tube was inserted and anesthesia was maintained by inhalation of $1.5-2.5 \%$ halothane, $\mathrm{N}_{2} \mathrm{O}$, and oxygen through the endotracheal tube. After a catheter was inserted into the left external jugular vein for administering drugs, sodium pentobarbital (30-40 mg/kg) was I.V. administered and the inhalation gas stopped. If the animal's arterial blood pressure and/or heart rate increased in response to a surgical procedure, and/or its pupils dilated, and/or it exhibited a withdrawal of the limb in response to a noxious pinch of the paw, additional sodium pentobarbital $(2-5 \mathrm{mg} / \mathrm{kg}$ I.V.) was administered to maintain an adequate depth of anesthesia.

A tracheotomy was performed and the trachea was directly intubated. Catheters were inserted into the left carotid artery for measuring arterial pressure (AP) and into the left femoral artery for withdrawing arterial blood. Heparin sodium was intravenously injected for anticoagulation. The carotid artery catheter was connected to a pressure transducer (DPT-III, Baxter). Mean arterial pressure (MAP) was obtained by integrating the arterial pressure signal with a time constant of $1 \mathrm{~s}$. The heart rate (HR) was derived from arterial pressure pulse by a tachometer (NEC San-ei, 1321).

A laminectomy was performed and the right $\mathrm{L}_{7}$ and $\mathrm{S}_{1}$ ventral roots were identified and cut. A pair of stimulating silver-wire electrodes $(0.1 \mathrm{~mm}$ in diameter) were implanted on the peripheral end of each sectioned ventral root. The stimulating electrodes were covered with a silicone gel for insulation, and the spinal incision was closed. The cat was placed in a lateral posture, and the pelvis and the right knee and ankle joints were clamped to prevent body movement during contraction. The right triceps surae muscle, calcaneous tendon, and calcaneous bone were exposed. The triceps surae muscle was isolated from surrounding connective tissue. The calcaneous tendon was severed from the calcaneous bone and attached to a force transducer (model LC1205-KC50, A\&D, 
Fig. 1. Experimental protocol. The five sequential interventions of the triceps surae muscle were performed throughout the experiment: (1) static contraction evoked by ventral root stimulation at $40 \mathrm{~Hz}$; (2) static contraction evoked by ventral root stimulation at $20 \mathrm{~Hz}$; (3) ventral root stimulation at $40 \mathrm{~Hz}$ with muscle paralysis; (4) mechanical stretch by $15 \mathrm{~mm}$ with muscle paralysis; (5) mechanical stretch by $10 \mathrm{~mm}$ with muscle paralysis. To induce static contraction, the $L_{7}$ and $S_{1}$ ventral roots were si-

multaneously stimulated (intensity, 1.5-2×motor threshold; duration $0.1-0.5 \mathrm{~ms}$ ). The developed tension during a $15 \mathrm{~mm}$ stretch corresponded to that during static contraction at $40 \mathrm{~Hz}$ ventral root stimulation; the developed tension during a $10 \mathrm{~mm}$ stretch corresponded to that during static contraction at $20 \mathrm{~Hz}$ ventral root stimulation. The duration of each trial was $1.5 \mathrm{~min}$. An intertrial rest was taken for more than $15 \mathrm{~min}$. After the end of the second trial, pancuronium bromide (1-2 mg I.M.) was administered to prevent muscle contraction. In each trial, heparinized arterial blood (sampled volume, 3-4 ml) was withdrawn before and at $1 \mathrm{~min}$ after the trial onset.

Tokyo) to measure the tension generated by the triceps surae muscle. To determine the optimal muscle length $\left(L_{0}\right)$ for producing maximal tension in the right triceps surae muscle, we induced a twitch static contraction by ventral root stimulation with a single shock. The muscle length at which the maximal twitch tension was obtained was defined as $L_{0}$ and used as the initial length of the triceps surae muscle throughout the experiment. The rectal temperature was continuously monitored throughout the experiment and maintained from 36 to $39^{\circ} \mathrm{C}$ by an external heating pad and a lamp. The animals were killed with an overdose of pentobarbital at the end of the experiments.

Experimental protocol. After more than $1 \mathrm{~h}$ for stabilization had been allowed, the experiment was started. As illustrated in Fig. 1, the five kinds of sequential interventions of the triceps surae muscle were performed in 10 cats: (1) static contraction evoked by ventral root stimulation at $40 \mathrm{~Hz}$; (2) static contraction evoked by ventral root stimulation at $20 \mathrm{~Hz}$; (3) ventral root stimulation at $40 \mathrm{~Hz}$ with muscle paralysis; (4) mechanical stretch by $15 \mathrm{~mm}$ with muscle paralysis; and (5) mechanical stretch by $10 \mathrm{~mm}$ with muscle paralysis. The initial muscle length was set at $L_{0}$. The $\mathrm{L}_{7}$ and $\mathrm{S}_{1}$ ventral roots were simultaneously stimulated to induce static contraction (intensity, $1.5-2 \times$ motor threshold; duration, $0.1-0.5 \mathrm{~ms}$ ). The developed tension during a $15 \mathrm{~mm}$ stretch corresponded to that during static contraction at $40 \mathrm{~Hz}$ ventral root stimulation; the developed tension during a $10 \mathrm{~mm}$ stretch corresponded to that during static contraction at $20 \mathrm{~Hz}$ ventral root stimulation. The duration of each trial was $1.5 \mathrm{~min}$. A rest between trials was taken for more than $15 \mathrm{~min}$. After the end of the second trial, pancuronium bromide (1-2 mg I.M.) was administered to prevent muscle contraction; the third, fourth, and fifth trials were conducted under neuromuscular blockade. The lungs were artificially ventilated with a mixture of room air and $\mathrm{O}_{2}$. The level of anesthesia was assessed by monitoring $\mathrm{AP}$ and $\mathrm{HR}$ after inducing muscle paralysis. If $\mathrm{AP}$ and/or $\mathrm{HR}$ increased in response to pain stimuli, supplemental pentobarbital $(2-5 \mathrm{mg} / \mathrm{kg}$ I.V.) was provided.

AP, mean arterial blood pressure (MAP), HR, and muscle tension were measured throughout the experiment. In each trial, heparinized arterial blood (sampled volume, 3-4 ml) was taken before and at $1 \mathrm{~min}$ after the trial onset. It took $15-20 \mathrm{~s}$ to withdraw the arterial blood. After each blood sample was immediately centrifuged, the plasma $(1.5-2 \mathrm{ml})$ was separated. The remaining blood cells and platelets were I.V. injected. After catecholamines were purified from plasma, a catecholamine assay was performed by reversed-phase high-pressure liquid chromatography with electrochemical detection according to our previous studies [7-9].

To examine whether the responses in plasma catecholamines to the muscle interventions were mainly derived from the adrenal medulla, we conducted the same kinds of muscle interventions after bilateral adrenalectomy in 5 cats. To perform adrenalectomy, we exposed the adrenal gland. All blood vessels and nerve bundles to the adrenal gland were tied and cut.

Statistical analysis. The changes in concentrations of plasma catecholamins [epinephrine (Ep) and norepinephrine (NEp)], MAP, HR, and muscle tension before and during muscle manipulation were measured and compared with the one-way analysis of variance (ANOVA) with repeated measurements. If the significant main effect was obtained, the multiple comparison between the mean value at a given time and the control value was performed by the "Dunnett" 
method. A statistical difference between the mean values of paired individual data was analyzed by the paired $t$-test. The level of the statistical significance was defined at $p<0.05$. The data are expressed as means \pm SEM.

\section{RESULTS}

\section{Reflex responses in MAP and HR to static contraction and to mechanical stretch}

Figure 2 shows the responses in muscle tension, MAP, and HR to ventral root stimulation and to mechanical stretch of the triceps surae muscle with and without muscle paralysis. Static contraction was induced by stimulation of the ventral roots at either 40 or $20 \mathrm{~Hz}$. The peak-developed tension during static contraction was $13.1 \pm 2.2 \mathrm{~kg}$ at $40 \mathrm{~Hz}$ and $6.6 \pm 1.2 \mathrm{~kg}$ at $20 \mathrm{~Hz}$. MAP and HR significantly increased from the baseline values of $130 \pm 5.0 \mathrm{mmHg}$ and $218 \pm 7.9$ beats/min during static contraction evoked by ventral

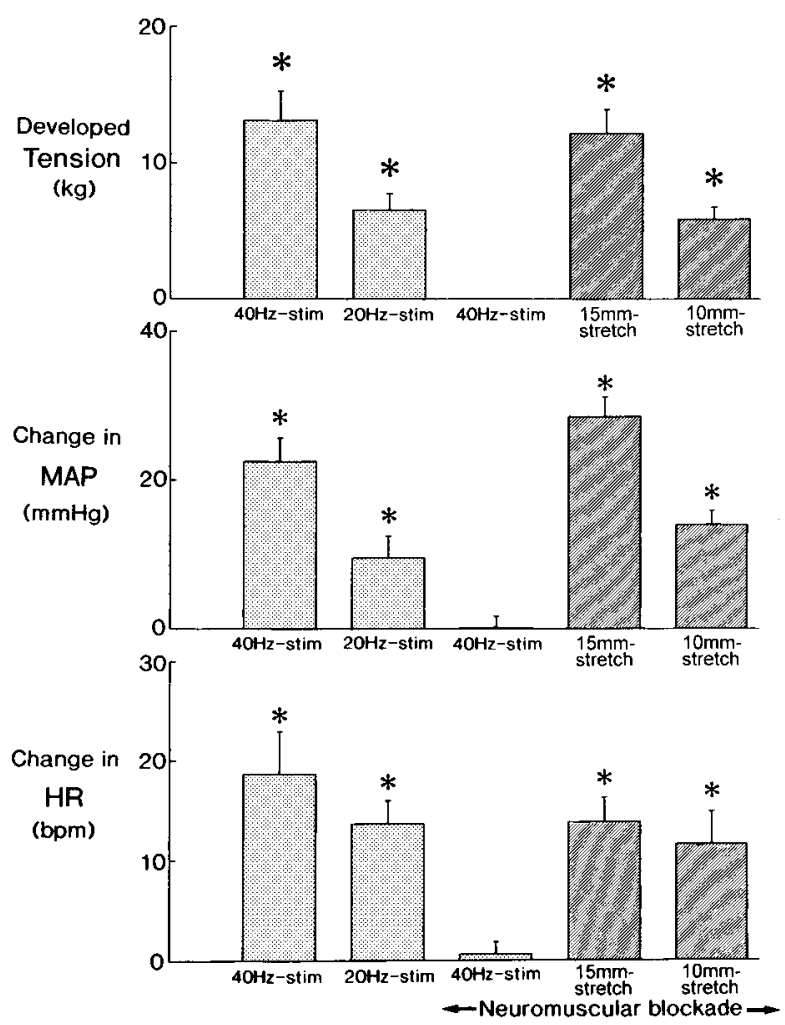

Fig. 2. The average responses in muscle developed tension, mean arterial pressure (MAP), and heart rate (HR) to ventral root stimulation and to mechanical stretch of the triceps surae muscle with and without muscle paralysis in $\mathbf{1 0}$ cats. Static contraction was induced by electrical stimulation of the ventral roots at either 40 or $20 \mathrm{~Hz}$. Following neuromuscular blockade, the same ventral root stimulation failed to increase MAP and HR. When the triceps surae muscle was stretched, however, MAP and HR could increase even after muscle paralysis. * Significant changes from the baseline control values. root stimulation (Fig. 2). The increases in MAP and HR were greater at $40 \mathrm{~Hz}$ ventral root stimulation than at $20 \mathrm{~Hz}$ ventral root stimulation $(23 \pm 3.1$ vs. $10 \pm 2.9$ $\mathrm{mmHg}$ and $19 \pm 4.3$ vs. $14 \pm 2.4$ beats/min). Following neuromuscular blockade, the baseline level of MAP decreased to $110 \pm 3.9 \mathrm{mmHg}$, but HR $(225 \pm 8.7$ beats/min) did not change from what it was before the neuromuscular blockade. The same ventral root stimulation failed to increase MAP and HR after neuromuscular blockade (Fig. 2). When the triceps surae muscle was stretched, however, MAP and HR could increase even after muscle paralysis (MAP, $29 \pm 2.6 \mathrm{mmHg}$ during a $15 \mathrm{~mm}$ stretch and $14 \pm 1.9 \mathrm{mmHg}$ during a $10 \mathrm{~mm}$ stretch; HR, $14 \pm 2.5$ beats/min during a $15 \mathrm{~mm}$ stretch and $12 \pm 3.3$ beats/min during a $10 \mathrm{~mm}$ stretch).

\section{Reflex responses in plasma catecholamines to static contraction and to mechanical stretch}

The absolute levels of plasma catecholamines during the muscle interventions throughout the experiments are demonstrated in Fig. 3. The changes in plasma catecholamines from the preceding baseline
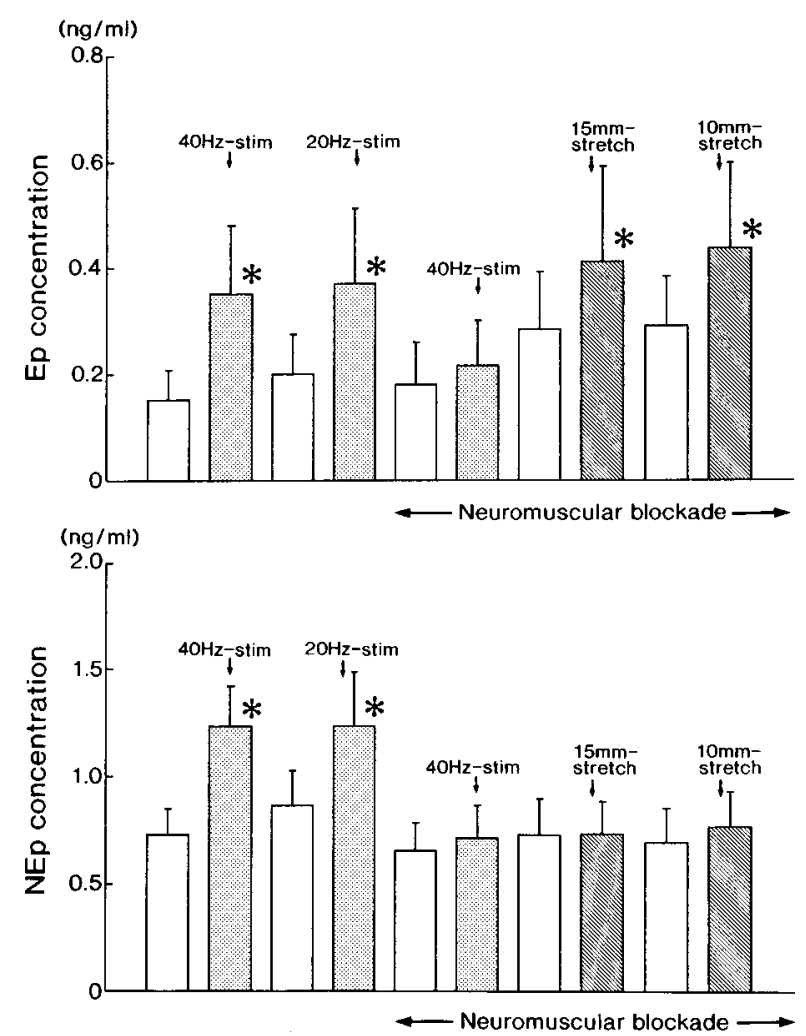

Fig. 3. Plasma catecholamines during static contraction evoked by ventral root stimulation (dotted columns) and during mechanical stretch (hatched columns) of the triceps surae muscle with and without neuromuscular blockade in $\mathbf{1 0}$ cats. Ep, epinephrine; NEp, norepinephrine. Open columns show the baseline control values. * Significant differences from the first baseline control values. 


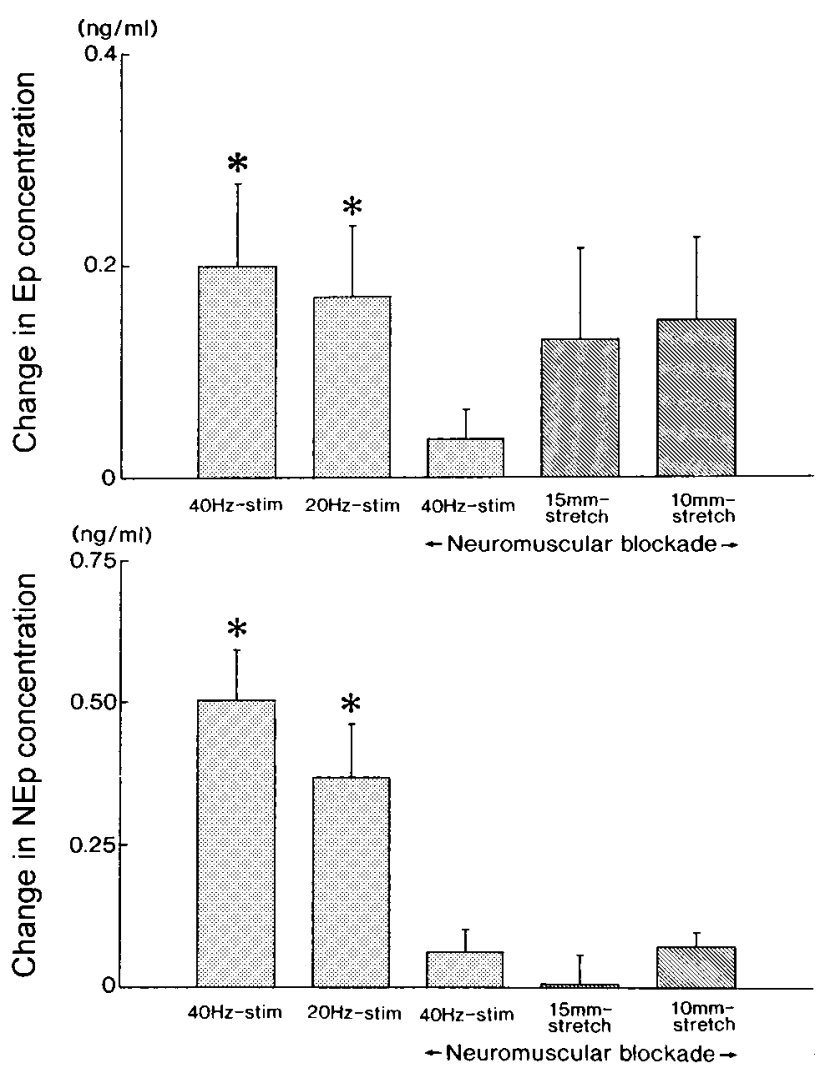

Fig. 4. The average responses in plasma Ep and NEp during static contraction evoked by ventral root stimulation (dotted columns) and during mechanical stretch (hatched columns) of the triceps surae muscle with and without neuromuscular blockade ( $n=10$ cats). * Significant changes from the baseline control values.

values, in response to each intervention, are summarized in Fig. 4. The baseline value of Ep was 0.14士 $0.051 \mathrm{ng} / \mathrm{ml}$; the baseline value of NEp was $0.71 \pm$ $0.108 \mathrm{ng} / \mathrm{ml}$. In Fig. 3, static contraction evoked by ventral root stimulation significantly increased plasma Ep $(0.18 \pm 0.072 \mathrm{ng} / \mathrm{ml}$ at $40 \mathrm{~Hz}$, and $0.16 \pm 0.061$ $\mathrm{ng} / \mathrm{ml}$ at $20 \mathrm{~Hz})$ and NEp $(0.47 \pm 0.087 \mathrm{ng} / \mathrm{ml}$ at $40 \mathrm{~Hz}$, and $0.35 \pm 0.085 \mathrm{ng} / \mathrm{ml}$ at $20 \mathrm{~Hz}$ ). Following neuromuscular blockade, the same ventral root stimulation failed to increase the concentrations of plasma catecholamines. A mechanical stretch of the muscle increased plasma Ep, but not plasma NEp in Fig. 3. The same tendency of the catecholamine responses to muscle intervention was seen in the paired data from the preceding control in Fig. 4.

\section{Effects of adrenalectomy on the plasma cate- cholamine responses}

To determine whether the responses in plasma catecholamines to static contraction and mechanical stretch of the triceps surae muscle were mainly derived from the adrenal medulla, we examined the effects of adrenalectomy on the plasma catecholamines
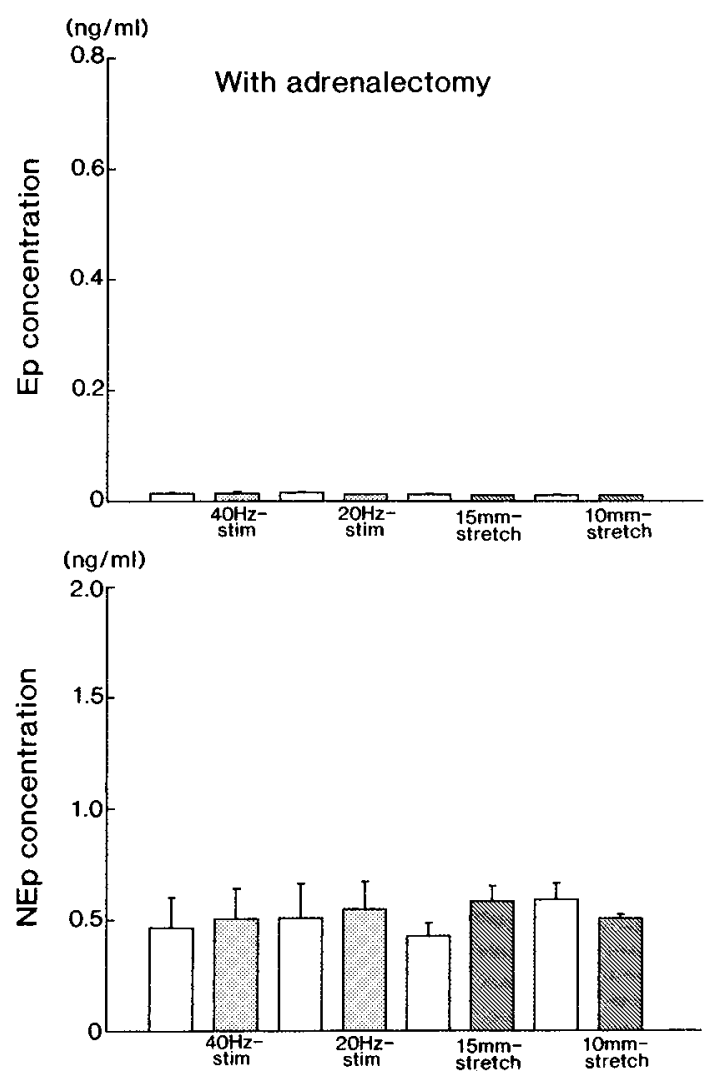

Fig. 5. The effects of bilateral adrenalectomy on plasma Ep and NEp during static contraction evoked by ventral root stimulation (dotted columns) and during mechanical stretch (hatched columns) of the triceps surae muscle in $\mathbf{5}$ cats. With the adrenalectomy, the baseline value of plasma Ep became negligible and the baseline NEp was slightly lowered. Neither Ep nor NEp in plasma changed significantly from the control values (open columns) during static muscle contraction or during mechanical stretch.

responses during the muscle interventions, as shown in Fig. 5. With the adrenalectomy, the baseline value of plasma Ep became negligible $(0.012 \pm 0.001 \mathrm{ng} / \mathrm{ml})$. The baseline NEp was also slightly lowered to $0.52 \pm$ $0.109 \mathrm{ng} / \mathrm{ml}$ by the adrenalectomy. Neither static muscle contraction nor mechanical stretch induced a significant response in plasma Ep. Furthermore, it is of interest that no significant responses in plasma NEp were produced during static contraction and during mechanical stretch. In accordance with the attenuated responses in plasma catecholamines, the increases in MAP and HR $(10 \pm 10.6 \mathrm{mmHg}$ and $14 \pm 2.7$ beats/ min) during static contraction at $40 \mathrm{~Hz}$ ventral root stimulation seemed to be reduced following adrenalectomy.

\section{DISCUSSION}

The reflex responses in plasma concentrations of cate- 
cholamines to static contraction of skeletal muscle have been investigated by the use of anesthetized cats. We found that static contraction of the triceps surae muscle evoked by ventral root stimulation increases plasma Ep 184\% and NEp 72\% within at least $1 \mathrm{~min}$ after the onset of contraction, which is abolished by muscle paralysis despite the identical ventral root stimulation. This result suggests that the responses in plasma catecholamines are caused by a reflex from the contracting muscle. Since most plasma Ep is derived from the adrenal medulla and its release rate is regulated by adrenal preganglionic sympathetic nerve activity (AdSNA), it is suggested that static contraction of skeletal muscle reflexly augments AdSNA, which secretes Ep and probably also NEp from the adrenal medulla into plasma. The present results are in good agreement with the previous data demonstrating a reflex increase in AdSNA during static muscle contraction in anesthetized rats [5].

With respect to the reflex adjustment of the sympathetic nervous system arising from the contracting muscles, static muscle contraction reflexly augments postganglionic sympathetic nerve activities to the heart, kidney, and skeletal muscle within $10 \mathrm{~s}$ from the onset of static muscle contraction evoked in anesthetized animals $[1-4,10]$. The rapid increase in cardiac sympathetic nerve activity contributes to an acceleration of cardiac rhythm [4], and the increase in renal sympathetic nerve activity contributes to renal vasoconstriction and a reduction in renal blood flow [3]. The initial cardiovascular responses can be explained by the reflex responses of postganglionic sympathetic nerve activities to the heart and blood vessels as well as a withdrawal of cardiac parasympathetic nerve activity [11], but not by a humoral effect of circulating catecholamines. The sustained cardiovascular responses during the later period of muscle contraction, however, may be explained by the humoral effect of circulating Ep secreted from the adrenal medulla in concert with the reflex responses of postganglionic sympathetic nerve activities. The increase in arterial plasma Ep to $0.33 \mathrm{ng} / \mathrm{ml}$ during muscle contraction obtained in this study is large enough to affect cardiac and vascular smooth muscles, because plasma Ep thresholds are as low as $0.05-0.2 \mathrm{ng} / \mathrm{ml}$ for its hemodynamic and metabolic actions in man [12]. On the other hand, it is known that arterial plasma NEp levels in excess of $1.8 \mathrm{ng} / \mathrm{ml}$ are required to produce hemodynamic and/or metabolic effects in man [13]. Although arterial plasma NEp increased to $1.2 \pm 0.23$ $\mathrm{ng} / \mathrm{ml}$ during static muscle contraction, it is very unlikely that the response imposes significant cardiovascular influences.
Vissing et al. [5] showed that AdSNA increases immediately after the onset of static muscle contraction in anesthetized rats, suggesting that a stimulation of muscle mechanoreceptors can augment AdSNA. In this study we found that plasma Ep is increased by mechanical stretch of skeletal muscle and by static muscle contraction. The increase in Ep observed during static contraction is therefore caused at least partly by a reflex increase in AdSNA because of a mechanical event of muscle contraction. Myelinated group III muscle afferents are quite likely to be excited by a mechanical event during muscle contraction, and unmyelinated group IV afferents are quite likely to be excited by a metabolic byproduct released during muscle contraction [6]. Taken together, the activation of myelinated group III mechanosensitive afferents during static contraction may stimulate AdSNA, which secretes epinephrine from the adrenal medulla.

In contrast to the plasma Ep response, NEp did not alter during a mechanical stretch of the triceps surae muscle. It is of interest that plasma Ep and NEp responded differently to the mechanical stretch. Assuming that the concentration of plasma NEp is an estimate of the total activity of the sympathetic nervous system [14], we find that this result suggests that the passive mechanical stretch muscle may not enhance the total activity of the sympathetic nervous system as much as static contraction does. Another interesting point is that the increases in MAP and HR were the same in spite of the difference in the response of plasma NEp. Taking the results into consideration, we think it is very likely that some specific organs (such as the heart, the kidney, and the adrenal medulla), but not all organs, are responsible for the cardiovascular responses during passive stretch, because cardiac and renal sympathetic nerve activities as well as plasma Ep are augmented during passive stretch [2-4].

It is believed that under resting conditions, the major source of NEp in plasma is postganglionic sympathetic nerve terminals and that only a small part (approximately 2\%) of NEp spillover into plasma is derived from the adrenal medulla [15], though nearly all plasma Ep is derived from it. Since the catecholamine output of the adrenal medulla was not directly measured in this study, the effects of bilateral adrenalectomy was performed to examine the adrenal medulla's role on the responses in arterial plasma catecholamines. As expected, the baseline plasma Ep and its responses to muscle contraction and to passive stretch were abolished by the adrenalectomy, indicating a predominant role of the adrenal medulla on the Ep responses. On the other hand, although the baseline NEp was slightly decreased following adrenalec- 
tomy in accordance with the previous data [15], the increase in plasma NEp during static contraction was markedly blunted by the adrenalectomy. The present result suggests that the reflex response in plasma NEp during muscle contraction is mainly derived from the adrenal medulla. This is supported by a previous study demonstrating that an increase in preganglionic adrenal sympathetic nerve activity during coronary occlusion has a significant positive relationship with rises in arterial plasma NEp and Ep, which are abolished by bilateral adrenalectomy [16]. It has been reported, however, that presynaptic $\beta$-adrenoceptors on sympathetic nerve terminals that have a facilitatory effect on NEp release from the sympathetic terminals may be activated by circulating Ep [17, 18]. If this is so, bilateral adrenalectomy would cause a generalized attenuation of the responses in plasma NEp. It was recently shown that the infusion of Ep may facilitate NEp overflow through prejunctional $\beta_{2}$-adrenoceptors during swimming exercise in adrenalectomized rats [19]. It is important to measure the NEp output from the adrenal medulla to determine the role of the adrenal medulla on the NEp response during static contraction.

This research was supported by a Grant-in-Aid for Scientific Research from the Ministry of Education, Science, Sports and Culture of Japan.

\section{REFERENCES}

1. Victor RG, Rotto DM, Pryor SL, and Kaufman MP: Stimulation of renal sympathetic nerve activity by static contraction: evidence for mechanoreceptor-induced reflexes from skeletal muscle. Circ Res 64: 592-599, 1989

2. Matsukawa K, Wall PT, Wilson LB, and Mitchell JH: Reflex responses of renal nerve activity during isometric muscle contraction in cats. Am $J$ Physiol 259: H1380-H1388, 1990

3. Matsukawa K, Wall PT, Wilson LB, and Mitchell JH: Neurally mediated renal vasoconstriction during isometric muscle contraction in cats. Am J Physiol 262: H833-H838, 1992

4. Matsukawa K, Wall PT, Wilson LB, and Mitchell JH: Reflex stimulation of cardiac sympathetic nerve activity during static muscle contraction in cats. Am J Physiol 267: H821-H827, 1994
5. Vissing J, Wilson LB, Mitchell JH, and Victor RG: Static muscle contraction reflexly increases adrenal sympathetic nerve activity in rats. Am $J$ Physiol 261: R1307-R1312, 1991

6. Mitchell $\mathrm{JH}$ : Neural control of the circulation during exercise. Med Sci Sports Exerc 22: 141-154, 1990

7. Honda T, Ninomiya I, and Azumi T: Cardiac sympathetic nerve activity and catecholamine kinetics in cat hearts. Am J Physiol 252: H879-H885, 1987

8. Matsukawa K, Honda T, and Ninomiya I: Renal sympathetic nerve activity and plasma catecholamines during eating in cats. Am J Physiol 257: R1034-R1039, 1989

9. Matsukawa K, Ninomiya I, and Nishiura N: Effects of anesthesia on cardiac and renal sympathetic nerve activities and plasma catecholamines. Am J Physiol 265: R792-R797, 1993

10. Hill JM, Adreani CM, and Kaufman MP: Muscle reflex stimulates postganglionic efferents innervating triceps surae muscles of cats. Am J Physiol 271: H38-H43, 1996

11. Murata J and Matsukawa K: Cardiac vagal and sympathetic efferent discharges are differentially modified by stretch of skeletal muscle. Am J Physiol Heart Circ Physiol 280: H237-H245, 2001

12. Clutter WE, Bier DM, Shah SD, and Cryer PE: Epinephrine plasma metabolic clearance rates and physiologic thresholds for metabolic and hemodynamic actions in man. J Clin Invest 66: 94-101, 1980

13. Silverberg AB, Shah SD, Haymond MW, and Cryer PE: Norepinephrine: hormone and neurotransmitter in man. Am J Physiol 234: E252-E256, 1978

14. Goldstein DS, McCarty R, Polinsky RJ, and Kopin IJ: Relationship between plasma norepinephrine and sympathetic nerve activity. Hypertension 5: 552-559, 1983

15. Esler M, Jennings G, Korner P, Willett I, Dudley F, Hasking $G$, Anderson W, and Lambert G: Assessment of human sympathetic nervous system activity from measurements of norepinephrine turnover. Hypertension 11: 3-20, 1988

16. Honda T and Ninomiya I: Changes in AdSNA and arterial catecholamines to coronary occlusion in cats. Am J Physiol 255: H704-H710, 1988

17. Misu $Y$ and Kubo T: Presynaptic beta-adrenoceptors. Med Res Rev 6: 197-225, 1986

18. Nedergaard $O A$ and Abrahamsen J: Modulation of noradrenaline release by activation of presynaptic betaadrenoceptors in the cardiovascular system. Ann N Y Acad Sci 604: 528-544, 1990

19. Coppes RP, Smit J, Benthem L, Van der Leest J, and Zaagsma J: Co-released adrenaline markedly facilitates noradrenaline overflow through prejunctional beta 2-adrenoceptors during swimming exercise. Eur J Pharmacol 274: 33-40, 1995 